\title{
A CHARACTERIZATION OF THE RIESZ SPACE OF MEASURABLE FUNCTIONS
}

\author{
BY \\ J. J. MASTERSON
}

1. Introduction. In [2], Kakutani has shown that any abstract $(L)$-space (Banach lattice such that $\|u+v\|=\|u\|+\|v\|$ for $u \geqq 0$ and $v \geqq 0$ ) can be represented as a space $L_{1}(X, \mathscr{S}, \mu)$ of equivalence classes of $\mu$-summable functions, where $\mu$ is a completely additive measure on a $\sigma$-algebra of subsets of some set $X$. This result can also be viewed as a characterization of the class of spaces $L_{1}(X, \mathscr{S}, \mu)$ by properties of the norm and order.

The purpose of this note is to show that an analogous classification exists for the class of spaces $\mathscr{M}(X, \mathscr{S}, \mu)$ (the set of equivalence classes of almost-everywhere finite valued $\mu$-measurable functions on the measure space $(X, \mathscr{S}, \mu))$ as long as $\mu$ is restricted to be a $\sigma$-finite measure. Since such spaces can not be normed, the characterization will involve only order properties. The above-mentioned classical theorem of Kakutani will be of significant value in obtaining our result.

The principal condition needed is a reflexivity property for Riesz spaces (vector lattices) which we will study in $\$ 2$. Lastly, we examine the non $\sigma$-finite case.

2. Riesz space preliminaries. Let $L$ be an Archimedean Riesz space throughout this paper. A linear functional $\varphi$ defined on $L$ is said to be a normal integral if $0 \leqq u_{\tau} \downarrow 0$ in $L$ implies that inf $\left|\varphi\left(u_{\tau}\right)\right|=0$. The space $L_{n}^{\tilde{n}}$ of normal integrals is a closed ideal in the order-bound dual of $L$. (Further explanation of the definitions and notations used may be found in [3].)

In [3], the concept of the normal integral is generalized by considering functionals that are normal integrals for some dense ideal $D \subset L$. This gives rise to an extended dual space which we denote by $\Gamma(L)$. The space $\Gamma(L)$ is a Dedekind complete and universally complete Riesz space, containing $L_{\tilde{n}}$ as an ideal [3, Theorems 2.5 and 2.6].

If $\Gamma(L)$ is separating on $L$, then $L$ is an order-dense Riesz subspace of $\Gamma^{2}(L)$. If $\Gamma^{2}(L)=L$, we say that $L$ is perfect in the extended sense. This is the reflexivity condition referred to in the introduction. The following result [3, Theorem 2.7] is a characterization which will be used:

(A) A Riesz space $L$, for which $\Gamma(L)$ is separating, is perfect in the extended sense if and only if $0 \leqq u_{\tau} \in L, u_{\tau} \uparrow$ and

$$
\sup \varphi\left(u_{\tau}\right)<\infty
$$

Received by the editors August 16, 1967. 
for every $0 \leqq \varphi \in \Gamma(L)$ which belongs to some order-dense ideal in $\Gamma(L)$, implies that $\sup \left(u_{\tau}\right)=u$ exists.

Notice that the condition $\Gamma^{2}(L)=L$ implies that $L$ is Dedekind complete but that in the general non $\sigma$-finite case, $\mathscr{M}(X, \mathscr{S}, \mu)$ need not be Dedekind complete. This gives rise to the restriction on the measure $\mu$.

We recall some définitions and facts about order completions of a Riesz space. $L$ is said to be universally complete if $0 \leqq u_{\tau} \in L$ and inf $\left(u_{\tau_{1}}, u_{\tau_{2}}\right)=0$ for $\tau_{1} \neq \tau_{2}$ imply that $u=\sup \left(u_{\tau}\right)$ exists in $L$. Nakano has shown [5] that every Archimedean Riesz space can be embedded as a dense Riesz subspace, in a Dedekind complete and universally complete Riesz space $L^{\prime}$ in such a way that suprema taken in $L$ are preserved when taken in $L^{\prime}$. The latter space is unique (up to isomorphism) and is called the universal completion of $L$. Moreover, if $L$ is Dedekind complete, then it is an ideal in $L^{\prime}$. From the uniqueness it follows that if $L$ is universally complete and Dedekind complete and $D$ is a dense ideal in $L$, then $L$ is the universal completion of $D$. Hence, if $\Gamma(L)$ is separating on $L$, implying $L$ is dense in $\Gamma^{2}(L)$, then $L$ is Dedekind complete and universally complete if and only if $L$ is perfect in the extended sense.

3. The characterization. Given any element $\psi$ in $\Gamma(L)$, there is a unique largest order-dense ideal to which $|\psi|$ can be extended finitely. We denote it by $D_{\psi}$. $\psi \mid D_{\psi}$ is a normal integral. Moreover, a positive element $u$ in $L$ is in $D_{\psi}$ if and only if

$$
\sup \left\{\psi(v): 0 \leqq v \leqq u, v \in I_{\psi}\right\}<\infty,
$$

where $I_{\psi}$ is any dense ideal on which $\psi$ is defined (that is, finitely defined) [3]. Moreover, Theorem 2.5 of the paper just cited says that if $\Gamma(L)$ is separating on $L$, there exists in $\Gamma(L)$ a strictly positive linear functional $\varphi$, that is, one such that $\varphi(u)>0$ for all $u \in D_{\varphi}, u>0$. We now state the central result.

THEOREM. Let L be a Riesz space which is Dedekind complete, universally complete and such that $\Gamma(L)$ is separating on $L$. Then $L$ contains a dense ideal which is an abstract (L)-space.

Proof. Let $\varphi$ be a strictly positive element in $\Gamma(L)$. Let $D$ be the (unique) largest order-dense ideal to which $|\varphi|$ can be extended finitely. For any $u$ in $L$ define $\|u\|=\varphi(|u|)$. Then $\|u\| \geqq 0$; equals zero if and only if $u=0$ since $\varphi$ is strictly positive. $\|a u\|=|a|\|u\|$ for a scalar $a$ and $\|u+v\| \leqq\|u\|+\|v\|$ follow from the linearity of $\varphi$. That $|u| \leqq|v|$ implies $\|u\| \leqq\|v\|$ results from the positivity of $\varphi$. So, with $\|\cdot\|$ as norm, $D$ is a normed vector lattice. If $u \geqq 0$ and $v \geqq 0,\|u+v\|=\varphi(|u+v|)=\varphi(u+v)$ $=\varphi(u)+\varphi(v)=\varphi(|u|)+\varphi(|v|)=\|u\|+\|v\|$. To show that $D$ is an abstract $(L)$-space then, it remains only to prove that $D$ is a complete space under $\|\cdot\|$.

Let $\left\langle u_{n}\right\rangle$ be an absolutely convergent series in $D$. So, there is $M$ such that $\sum_{n=1}^{\infty}\left\|u_{n}\right\| \leqq M<\infty$. But then $\left\|u_{n}^{+}\right\| \leqq\left\|u_{n}\right\|$ and $\left\|u_{n}^{-}\right\| \leqq\left\|u_{n}\right\|$ imply that $\left\langle u_{n}^{+}\right\rangle$and $\left\langle u_{n}^{-}\right\rangle$are absolutely convergent series in $D$ with the same absolute bound $M$. 
Let $v_{n}=\sum_{k=1}^{n} u_{k}^{+}$. Then, $\left\langle v_{n}\right\rangle$ is an increasing sequence in $D$ and $\left\|v_{n}\right\| \leqq M, n=1$, $2, \ldots$ So, $\varphi\left(v_{n}\right) \leqq M, n=1,2, \ldots$ and hence $\sup _{n} \varphi\left(v_{n}\right)<\infty$. For any $\psi$ in $I$, then, where $I$ is the principal ideal generated by $\varphi$, we have

$$
\sup _{n} \psi\left(v_{n}\right)<\infty
$$

However, since $\varphi$ is strictly positive, $I$ is an order-dense ideal in $\Gamma(L)$. Hence, the conditions in the conclusion of (A) are satisfied for the sequence $\left\{v_{n}\right\}$. Since $L$ is perfect in the extended sense (being Dedekind complete and universally complete) then, $v=\sup \left(v_{n}\right)$ must exist in $L$. Now, $\sup \varphi\left(v_{n}\right) \leqq M$ implies that

$$
\sup \{\varphi(w): 0 \leqq w \leqq v, w \in D\}<\infty .
$$

(For if $0 \leqq w \leqq v, \sup \left(\inf \left(w, v_{n}\right)\right)=w$, hence $\varphi(w) \leqq M$.) By remarks made above, then, $v$ is in $D$, and since $\varphi$ is a normal integral on $D, \lim \varphi\left(v_{n}\right)=\varphi(v)$. So, $\lim \left\|v-v_{n}\right\|=0$, hence $\lim \left\|v-\sum_{k=1}^{n} u_{k}^{+}\right\|=0$. In the same way, we obtain $v^{\prime}$ in $D$ such that $\lim \left\|v^{\prime}-\sum_{k=1}^{n} u_{k}^{-}\right\|=0$. But then, $\lim \left\|\left(v-v^{\prime}\right)-\sum_{k=1}^{n} u_{k}\right\|=0$. We have shown that every absolutely summable series in $D$ is summable. Hence, $D$ is complete, finishing the proof.

Using Kakutani's representation theorem, the ideal $D$ is isomorphic to a space $L_{1}=L_{1}(X, \mathscr{S}, \mu)(\mu$ may not be $\sigma$-finite). Let $\mathscr{M}=\mathscr{M}(X, \mathscr{S}, \mu)$ be the associated space of equivalence classes of measurable functions. Now $D$ (or $L_{1}$ ) may be regarded as a dense ideal in both $\mathscr{M}$ and $L$. Hence, using [3, Theorems 2.6 and 2.3] (along with the hypotheses of the theorem) we have $\mathscr{M} \subset \Gamma^{2}(\mathscr{M})=\Gamma^{2}(L)=L$. Moreover, $L$ is the universal completion of $\mathscr{M}$. So,

Corollary 1. Let L be a Riesz space which is Dedekind complete, universally complete and such that $\Gamma(L)$ is separating on $L$. Then, $L$ contains a dense Riesz subspace which is isomorphic to $\mathscr{M}(X, \mathscr{S}, \mu)$.

The Riesz space $L$ is said to be super Dedekind complete if $L$ is Dedekind complete and if any subset $A \subset L$ which is bounded above contains an, at most, countable subset having the same least upper bound as the whole set $A$.

This property was introduced by Luxemburg and Zaanen in [4] and studied in their later papers under the same title.

It can now be shown that if this condition is added to the hypotheses of the theorem, the measure $\mu$ obtained in the corollary above must be $\sigma$-finite.

Let $\left\{X_{\alpha}\right\}$ be the class of all sets of finite $\mu$-measure. We must have then, that $\chi_{X}=\sup \left(\chi_{X_{\alpha}}\right)$ in $L$ ( $\chi_{E}$ being the class containing the characteristic function of $E$ ). There must then be an at most countable subset $\left\{\chi_{x_{n}}\right\}$ such that $\chi_{X}=\sup \left(\chi_{x_{n}}\right)$. But then $X=\bigcup_{n=1}^{\infty} X_{n}$ (except possibly for a set of $\mu$ measure zero). Hence, $\mu$ is a $\sigma$-finite measure on $S$.

With $\mu \sigma$-finite, we can show that $\mathscr{M}=L$. First of all, if $\mu$ is $\sigma$-finite, then $\mathscr{M}$ is Dedekind complete. [1, IV, p. 335.] The following two facts are also readily obtained 
(a) $\mathscr{M}$ contains a complete element i.e., there is $0 \leqq e \in \mathscr{M}$ such that inf $(e, x)=0$ implies $x=0$.

(b) $\mathscr{M}$ contains the supremum of any countable disjoint set of its elements.

Nakano has shown [5] that for a Dedekind complete Riesz space, (a) and (b) are necessary and sufficient for universal completeness. So, $\mathscr{M}$ is universally complete and hence, $\mathscr{M}=L$.

We have obtained one half of the characterization described in the introduction. The other half is a rather routine verification. We state the result:

CoRollary 2. Let L be a Riesz space. In order that there exist a completely additive $\sigma$-finite measure space $(X, \mathscr{S}, \mu)$ such that $L=\mathscr{M}(X, \mathscr{S}, \mu)$, the following conditions are necessary and sufficient:

(1) $\Gamma(L)$ is separating on $L$,

(2) $L$ is universally complete,

(3) $L$ is super Dedekind complete.

4. The non $\sigma$-finite case. The last corollary cannot be modified to include any measure $\mu$, since even the condition that $\Gamma(L)$ be separating restricts the measure $\mu$ to have the finite subset property, that is, any set of $\mu$-positive measure has a subset of $\mu$-finite measure (a fact we used in proving the last corollary).

As can be seen from the first two corollaries, a characterization in the non $\sigma$-finite case will obtain if a condition on $\mu$ can be found which guarantees that $\mathscr{M}(X, \mathscr{S}, \mu)$ is Dedekind complete. Such a condition appears in a paper of A. C. Zaanen [6] concerning an extension of the Radon-Nikodym Theorem. Namely, the measure $\mu$ is said to be localizable if the lattice of equivalence classes of $\mu$ measurable sets is complete. (The term is originally due to I. E. Segal.) We discuss a characterization of this property.

Given a set $E$ of finite $\mu$-measure, let $M_{E}^{*}$ be the collection of equivalence classes $f^{*}$ of $\mu$-measurable functions $f$ vanishing almost everywhere off $E$. If for each such $E$ we select $f_{E}^{*}$ in $M_{E}^{*}$ in such a way that for $E$ and $F$ of finite measure we have $\left(f_{E \cap F}\right)^{*}=\left(f_{E} \chi_{E \cap F}\right)^{*}=\left(f_{F} \chi_{E \cap F}\right)^{*}$, then the collection $\left\{f_{E}^{*}\right\}$ is called a cross-section of $X$. Theorem 9.4 in [6] states that the measure $\mu$ is localizable if and only if for every cross-section $\left\{f_{E}^{*}\right\}$, there is a measurable $f$ defined on all of $X$ such that $\left(f \chi_{E}\right)^{*}=f_{E}^{*}$ for all $E$ of finite measure.

The value of localizability is that it characterizes those measure spaces for which there is a Radon-Nikodym Theorem.

Assuming now that $\mu$ is localizable, let $\left\{f_{\alpha}^{*}\right\}$ be a collection of nonnegative elements in $\mathscr{M}=\mathscr{M}(X, \mathscr{S}, \mu)$, bounded above by $g$. Then, $\left(f_{\alpha} \chi_{E}\right)^{*}$ is in $M_{E}^{*}$ for each set $E$ of finite measure. But, $M_{E}^{*}$ is Dedekind complete since the restriction of $\mu$ to $E$ is a finite measure. Moreover, $\left(f_{\alpha} \chi_{E}\right)^{*} \leqq\left(g \chi_{E}\right)^{*}$. Hence, $\sup \left(f_{\alpha} \chi_{E}^{*}\right)=f_{E}^{*}$ (supremum taken in $\mathscr{M}$ since $M_{E}^{*}$ is an ideal in $\mathscr{M}$ ) exists and is an element of $M_{E}^{*}$. Now it is easily verified that the collection $\left\{f_{E}^{*}\right\}$ is a cross-section, and hence there exists $f^{*}$ in $\mathscr{M}$ such that $\left(f \chi_{E}\right)^{*}=f_{E}^{*}$ for each set $E$ of finite measure. This last 
statement is true, however, only if $f^{*}=\sup \left(f_{\alpha}^{*}\right)$. So, $\mathscr{M}$ is complete and we have the following:

COROLlary 3. Let L be a Riesz space. The following conditions are necessary and sufficient that there exist a completely additive localizable measure $\mu$ such that $L=\mathscr{M}(X, \mathscr{S}, \mu)$ :

(1) $\Gamma(L)$ is separating,

(2) $L$ is Dedekind complete and universally complete.

\section{REFERENCES}

1. N. Dunford and J. T. Schwartz, Linear operators, Part I, Interscience, New York, 1958.

2. S. Kakutani, Concrete representation of abstract $(L)$-space and the mean ergodic theorem, Ann. of Math. 42 (1941), 523-537.

3. W. A. J. Luxemburg and J. J. Masterson, An extension of the concept of the order dual of a Riesz space, Canad. J. Math. 19 (1967), 488-498.

4. W. A. J. Luxemburg and A. C. Zaanen, Notes on Banach function spaces. VII, Nederl. Akad. Wetensch. Proc. Ser. A 66 (1963), 669-681.

5. H. Nakano, Modern spectral theory, Maruzen, Tokyo, 1950.

6. A. C. Zaanen, The Radon-Nikodym theorem. II, Nederl. Akad. Wetensch. Proc. Ser. A 64 (1961), 171-187.

\section{Michigan STATE UNiversity,}

East Lansing, Michigan 